\title{
Quasi-Vector Model of Propagation of Polarized Light in a Thin-Film Waveguide Lens
}

Dmitriy Divakov ${ }^{1, \star}$, Mikhail Malykh$^{1}$, Leonid Sevastianov ${ }^{1,2}$, Anton Sevastianov $^{1}$, and Edik Ayryan²

${ }^{1}$ Peoples' Friendship University of Russia (RUDN University), 117198, Moscow, Russia

${ }^{2}$ Joint Institute for Nuclear Research, 141980, Dubna, Moscow region, Russia

\begin{abstract}
Maxwell equations describe the propagation with diffraction of waveguide modes through a thin-film waveguide lens. If the radius of the thin-film lens is large, then the thickness of the lens varies slowly in the $y z$ plane. For this case we propose the model, which is based on the assumption of a small change in the electromagnetic field in a direction $y$. Under this assumption the vector diffraction problem is reduced to a number of scalar diffraction problems. The solutions demonstrate the vector nature of the electromagnetic field, which allows us to call the proposed model a quasi-vector model.
\end{abstract}

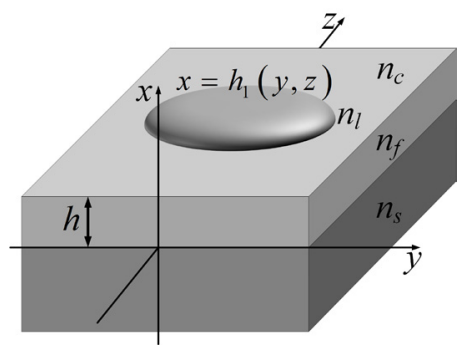

Figure 1. Thin-film waveguide lens of radius $R_{l}$ with varible height $h_{1}(y, z)$

\section{Introduction}

The first and the second Maxwell equations in the component representation in the Cartesian coordinate system for time-harmonic fields have the following form [1]:

$$
\begin{array}{ccc}
\frac{\partial H_{z}}{\partial y}-\frac{\partial H_{y}}{\partial z}=-i k_{0} \varepsilon E_{x}, & \frac{\partial H_{x}}{\partial z}-\frac{\partial H_{z}}{\partial x}=-i k_{0} \varepsilon E_{y}, & \frac{\partial H_{y}}{\partial x}-\frac{\partial H_{x}}{\partial y}=-i k_{0} \varepsilon E_{z}, \\
\frac{\partial E_{z}}{\partial y}-\frac{\partial E_{y}}{\partial z}=i k_{0} H_{x}, & \frac{\partial E_{x}}{\partial z}-\frac{\partial E_{z}}{\partial x}=i k_{0} H_{y}, & \frac{\partial E_{y}}{\partial x}-\frac{\partial E_{x}}{\partial y}=i k_{0} H_{z} .
\end{array}
$$

^e-mail: divakov_dv@rudn.university 


\section{Zeroth order approximation}

If the waveguide lens has a radius $R_{l} \gg \lambda$, where $\lambda$ is the wavelength, then $\left|\partial h_{1} / \partial y\right| \ll 1$ and assuming in this case that the electromagnetic field also varies slowly along $y$, we introduce a small parameter defined as $\delta=\max \left\{\left|\partial E_{\alpha} / \partial y\right|,\left|\partial H_{\alpha} / \partial y\right|, \quad \alpha=x, y, z\right\}$. In the zeroth order approximation over the small parameter $\delta$ the equations (1), (2) take the following form [2, 3]:

$$
\begin{aligned}
& \frac{\partial H_{x}^{(0)}}{\partial z}-\frac{\partial H_{z}^{(0)}}{\partial x}=-i k_{0} n^{2} E_{y}^{(0)}, \quad i k_{0} H_{x}^{(0)}=-\frac{\partial E_{y}^{(0)}}{\partial z}, \quad i k_{0} H_{z}^{(0)}=\frac{\partial E_{y}^{(0)}}{\partial x}, \\
& \frac{\partial E_{x}^{(0)}}{\partial z}-\frac{\partial E_{z}^{(0)}}{\partial x}=i k_{0} H_{y}^{(0)}, \quad i k_{0} n^{2} E_{x}^{(0)}=\frac{\partial H_{y}^{(0)}}{\partial z}, \quad-i k_{0} n^{2} E_{z}^{(0)}=\frac{\partial H_{y}^{(0)}}{\partial x},
\end{aligned}
$$

where $n(x, y, z)$ is the refractive index of the waveguide. The system of equations (1), (2) is now represented as two independent subsystems: the subsystem (3) for TE-mode and (4) for TM-mode, each subsystem can be represented as one equation of the second order for the leading component $\left(E_{y}\right.$ for TE-mode and $H_{y}$ for TM-mode) and two equations relating the remaining components through the leading one [1]. We consider the problem of waveguide diffraction of one $\mathrm{TE}_{0}$-mode incident on irregularity [2]. The formulation of the problem in the zeroth order approximation has the form [3]:

$$
\left\{\begin{array}{l}
\left(\Delta_{x, z}+k_{0}^{2} n^{2}(x, y, z)\right) E_{y}^{(0)}=0, \\
\left.E_{y}^{(0)}\right|_{z<-R_{l}}=A(y) e^{i k_{0} \beta_{0} z} \varphi_{0}(x)+\sum_{j=1}^{N} R_{j}^{\mathrm{TE}}(y) e^{-i k_{0} \beta_{j} z} \varphi_{j}(x), \\
\left.E_{y}^{(0)}\right|_{z>R_{l}}=\sum_{j=1}^{N} T_{j}^{\mathrm{TE}}(y) e^{i k_{0} \beta_{j} z} \varphi_{j}(x), \quad E_{y}^{(0)} \underset{x \rightarrow \pm \infty}{\rightarrow} 0,
\end{array}\right.
$$

where $\Delta_{x, z}=\partial^{2} / \partial x^{2}+\partial^{2} / \partial z^{2}, R_{j}^{\mathrm{TE}}(y)$ and $T_{j}^{\mathrm{TE}}(y)$ are reflection and transmission coefficients of the component $E_{y}$ (complex values, as in [4]), $A(y)$ determines the amplitude of the waveguide mode incident on the lens, $R_{l}$ is radius of the waveguide lens. The eigenvalues $\beta_{j}$ and eigenfunctions $\varphi_{j}(x)$ determine the TE-modes of the regular waveguide $\left(z>R_{l}, z<-R_{l}\right)$. The components $H_{x}^{(0)}$ and $H_{z}^{(0)}$ are expressed in terms of $E_{y}^{(0)}$ by means of (3). In addition, there is no TM-mode in the incident radiation, which leads us in the zeroth order approximation to $H_{y}^{(0)}=E_{x}^{(0)}=E_{z}^{(0)} \equiv 0$, therefore the reflection and transmission coefficients of the components $E_{x}^{(0)}$ and $E_{z}^{(0)}$ are zeros.

We seek the approximate solution $\tilde{E}_{y}^{(0)}$ of the problem (5) in the form: $\tilde{E}_{y}^{(0)}=\sum_{j=1}^{N} V_{j}(y, z) \varphi_{j}(x)$ where $V_{j}(y, z)$ are the desired functions [2,3,5]. We substitute this solution into (5) and perform the Galerkin method procedure [2]. As a result we obtain the mixed boundary value problem with respect to the desired Kantorovich coefficient functions $V_{j}(y, z)[3,5,6]$ :

$$
\left\{\begin{array}{l}
\vec{v}_{z z}^{\prime \prime}+\mathbf{Q}(y, z) \vec{v}=\overrightarrow{0}, \\
\left(\vec{v}_{z}^{\prime \prime}+i k_{0} \mathbf{D} \vec{v}\right)_{z=-R_{l}}=2 i k_{0} \mathbf{D} \vec{a}_{n_{0}}, \quad\left(\vec{v}_{z}^{\prime}-i k_{0} \mathbf{D} \vec{v}\right)_{z=R_{l}}=\overrightarrow{0} .
\end{array}\right.
$$

For each $y=y_{m}$, the problem (6) is a boundary value problem for a system of ordinary differential equations, in spite of the fact that the coefficient functions $V_{j}(y, z)$ depend on two variables. Differentiation is carried out only on the fast variable $z$ and the dependence on the slow variable $y$ is parametric.

Solving (5), we define the leading component $E_{y}^{(0)}$ and the components $H_{x}^{(0)}$ and $H_{z}^{(0)}$ of the TEmode by the formula (3). The remaining components corresponding to the TM-mode will be zero. The zeroth order approximation, therefore, does not describe the process of hybridization of modes and we now proceed to the first order approximation. 


\section{First order approximation}

We get the Maxwell equations in the first order approximation: $\vec{E}=\vec{E}^{(0)}+\vec{E}^{(1)}, \vec{H}=\vec{H}^{(0)}+\vec{H}^{(1)}$ considering that $\partial E_{\alpha}^{(1)} / \partial y, \partial H_{\alpha}^{(1)} / \partial y$ for $\alpha=x, y, z$ have the second order of smallness. We obtain the following equations for the TE-mode:

$$
\left(\Delta_{x, z}+k_{0}^{2} n^{2}(x, y, z)\right) E_{y}^{(1)}=0, \quad H_{x}^{(1)}=-\frac{1}{i k_{0}} \frac{\partial E_{y}^{(1)}}{\partial z}, \quad H_{z}^{(1)}=\frac{1}{i k_{0}} \frac{\partial E_{y}^{(1)}}{\partial x} .
$$

The formulation of the diffraction problem for the TE-mode in the first order approximation has the following form:

$$
\left\{\begin{array}{l}
\left(\Delta_{x, z}+k_{0}^{2} n^{2}(x, y, z)\right) E_{y}^{(1)}=0, \\
\left.E_{y}^{(1)}\right|_{z<-R_{l}}=\sum_{j=1}^{N} R_{j}^{(1)}(y) e^{-i k_{0} \beta_{j} z} \varphi_{j}(x),\left.\quad E_{y}^{(1)}\right|_{z>R_{l}}=\sum_{j=1}^{N} T_{j}^{(1)}(y) e^{i k_{0} \beta_{j} z} \varphi_{j}(x), \quad E_{y}^{(1)} \underset{x \rightarrow \pm \infty}{\rightarrow} 0 .
\end{array}\right.
$$

In the boundary conditions of the problem (8), which determine the reflected and transmitted fields, the incident field is absent, since it is completely taken into account in the zeroth order approximation, and in the first approximation gives only the first-order corrections to the reflection and transmission coefficients. Applying the incomplete Galerkin method to the diffraction problem (8), we obtain a boundary value problem for a homogeneous system of second-order differential equations with homogeneous boundary conditions of the third kind, which has only a trivial solution [2]. Thus, the correction of the first order $E_{y}^{(1)}$ vanishes identically and also $H_{x}^{(1)}=H_{z}^{(1)}=0$. The first-order correction $H_{y}^{(1)}$ is also zero. Corrections of the first order of smallness to the remaining components of the field are equal, respectively to:

$$
E_{x}^{(1)}=\frac{-1}{i k_{0} n^{2}} \frac{\partial H_{z}^{(0)}}{\partial y}, \quad E_{z}^{(1)}=\frac{1}{i k_{0} n^{2}} \frac{\partial H_{x}^{(0)}}{\partial y} .
$$

It follows from (9) that the TE-mode incident on the irregularity excites the components of the TMmode of the first order of smallness, that is, the solution obtained in the first approximation describes the hybridization of waveguide modes, which demonstrates the vector nature of the model.

\section{Numerical experiment}

We solve numerically the problem of waveguide diffraction of monochromatic light in the waveguide lens with the following input data: $n_{c}=1.0, n_{s}=1.47, n_{f}=1.565$ and $n_{l}=1.9$. The thickness of the waveguide layer is $2 \lambda$ where $\lambda=0.55 \mu$ is the wavelength. The waveguide mode $\mathrm{TE}_{0}$ with a unit amplitude $(A(y)=1)$ incidents on a waveguide lens with a radius $R_{l}=20 \lambda$. We consider the transmission coefficient of the component $E_{y}^{(0)}$ of the second waveguide mode, which was excited by the energy redistribution between modes during the propagation of the mode $\mathrm{TE}_{0}$. The order of smallness of $T_{2}^{\mathrm{TE}}(y)$, as follows from the Figure 2, corresponds to the assumption that the value of $\partial E_{y}^{(0)} / \partial y$ is small. The remaining components of the field have the transmission coefficients similar of the same order of smallness.

The transmission coefficients of the components $E_{x}^{(1)}$ and $E_{z}^{(1)}$ (see Fig. 3) are by an order smaller than the coefficient of transmission of $E_{y}^{(0)}$, which corresponds to the first approximation of the problem under consideration. The corrections of the first order of smallness to the remaining components of the field are zeros. 


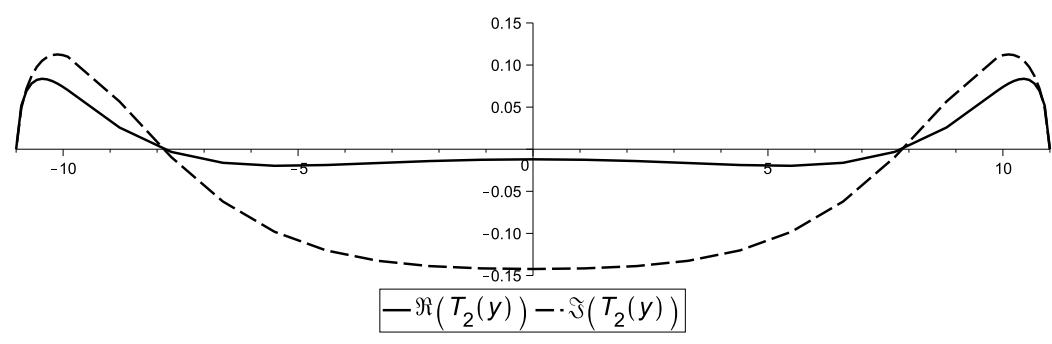

Figure 2. Transmission coefficient $T_{2}^{\mathrm{TE}}(y)$ of the component $E_{y}^{(0)}$ of the mode $\mathrm{TE}_{1}$

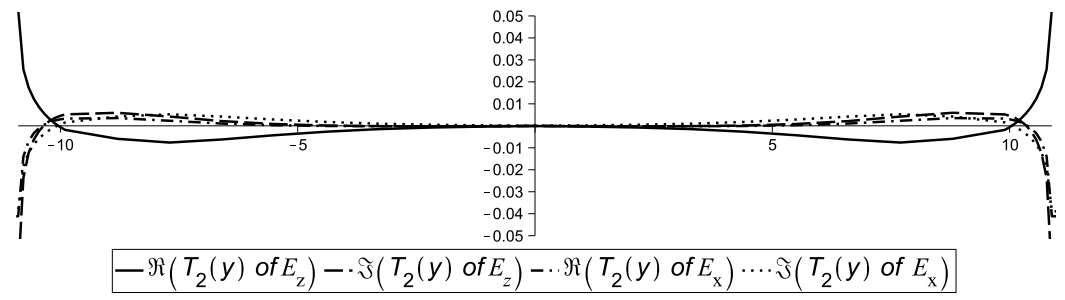

Figure 3. Transmission coefficient $T_{2}^{\mathrm{TM}}(y)$ of the components $E_{z}^{(1)}$ and $E_{x}^{(1)}$ of the mode $\mathrm{TM}_{1}$

\section{Conclusion}

The paper describes a quasi-vector model of propagation of waveguide modes through a thin-film waveguide lens of large radius. Under the assumption of a small change in the electromagnetic field with respect to $y$, one can construct a perturbation theory with respect to the corresponding small parameter. The proposed model allows us to formulate in the zeroth order approximation a series of scalar diffraction problems the TE-mode, the solutions of $m$-th problem describe the TE-mode evolution in the longitudinal section $y=y_{m}$. In the first approximation, these solutions are used to calculate the corrections $E_{x}^{(1)}, E_{z}^{(1)}$ perturbating the components of the TE- and TM-modes. Thus, as a result of solving a series of scalar problems, we obtain a solution that possesses the properties of the solution of the vector problem $\vec{E}=\left(E_{x}^{(1)}, E_{y}^{(0)}, E_{z}^{(1)}\right), \vec{H}=\left(H_{x}^{(0)}, 0, H_{z}^{(0)}\right)$.

\section{Acknowledgements}

The publication was prepared with the support of the "RUDN University Program 5-100". This research was supported by RFBF grants No 15-07-08795, No 16-07-00556.

\section{References}

[1] E. Ayryan et al., Proc. of SPIE 10337, 103370I (2017)

[2] A.G. Sveshnikov, Comp. Math and Math. Phys. 2, 1, 186-190 (1963)

[3] M.D. Malykh et al., RUDN Journal of Math., Inf. Sci. and Phys. 25:1, 56-68 (2017)

[4] A.G. Sveshnikov and A.S. Il'inskii, Zh. vych. mat. 3:3, 478-488 (1963)

[5] L.V. Kantorovich and V.I. Krylov, Approximate Methods of Higher Analysis (Wiley, New-York, 1964)

[6] D.V. Divakov, L.A. Sevastianov, and N.E. Nikolaev, EPJ Web of Conf. 108, 02020 (2016) 